\title{
Colored Noise in Quantum Chaos
}

\author{
Luca Salasnich \\ Istituto Nazionale per la Fisica della Materia, Unità di Milano Università \\ Dipartimento di Fisica, Università di Milano, \\ Via Celoria 16, 20133 Milano, Italy
}

\begin{abstract}
We derive a set of spectral statistics whose power spectrum is characterized, in the case of chaotic quantum systems, by colored noise $1 / f^{\gamma}$, where the integer parameter $\gamma$ critically depends on the specific energy-level statistic considered. In the case of regular quantum systems these spectral statistics show $1 / f^{\gamma+1}$ noise.
\end{abstract}

PACS Numbers: 05.45.Mt, 89.75.Da, 05.40.Ca

Quantum chaos is the study of quantum systems which are classically chaotic [1]. There is a conjectured relationship, backed up by numerous examples, between the energy level fluctuation properties of a quantum systems and the dynamical behavior of its classical analog. In particular, Berry and Tabor showed that classically integrable systems are characterized by Poissonian spectral fluctuations [2], while Bohigas, Giannoni and Schmit [3] suggested that classically chaotic systems can be described by Gaussian ensembles of random matrix theory (RMT). In the past years many energy-level statistics have been proposed to characterize the spectral fluctuations; among them there are the nearest-neighbour levelspacing distribution $P(s)$, the spectral rigidity $\Delta_{3}(L)$ and the spectral form factor $K(\tau)$. Recently, Relano et al. [4] have introduced the energy-level statistic $\delta_{n}$, showing that chaotic quantum systems display $1 / f$ noise in the $\delta_{n}$ statistic, whereas integrable ones exibit $1 / f^{2}$ noise $[4,5]$.

In this Brief Report we introduce a new set of spectral statistics which contains the spectral statistic $\delta_{n}$ as a particular case. We show that for chaotic quantum systems these statistics are characterized by colored noise $1 / f^{\gamma}$, where the integer parameter $\gamma$ critically depends on the specific energy-level statistic considered. Moreover, we find that in the case of regular quantum systems these spectral statistics display $1 / f^{\gamma+1}$ noise.

Let us consider a quantum system with a discrete set of unfolded levels $E_{n}$, such that the energy is measured in units of the mean level speacing. The staircase function $N(E)$, which gives the number of energy levels up to the energy $E$, can be written as

$$
N(E)=\bar{N}(E)+N_{o s c}(E)
$$

where $\bar{N}(E)=E$ is the averaged number of levels and $N_{\text {osc }}(E)$ is the fluctuating part. $N_{o s c}(E)$ is supposed to belong to a universality class, which should only depend on the integrability or chaoticity of the classical analog [1]. The Madrid group of Relano et al. [4] has analyzed these fluctuations by using the spectral statistic $\delta_{n}$, defined as

$$
\delta_{n}=N_{o s c}\left(E_{n}\right)
$$

In particular, the Madrid group has investigated the behavior of the power spectrum $S(k)$ of the discrete series $\delta_{n}$, given by

$$
S_{M}(k)=\left|\hat{\delta}_{k}\right|^{2},
$$

where $\hat{\delta}_{k}$ is the Fourier transform of $\delta_{n}$,

$$
\hat{\delta}_{k}=\frac{1}{\sqrt{M}} \sum_{n=1}^{M} \delta_{n} \exp \left(\frac{-i k n}{M}\right)
$$

and $M$ is the size of the series. By studying spectra of atomic nuclei at high energies and also spectra of Gaussian ensebles of RMT, the Madrid group has found the power law $\left\langle S_{M}(k)\right\rangle \sim 1 / k$. For Poisson spectra it has found instead $\left\langle S_{M}(k)\right\rangle \sim 1 / k^{2}$, as expected for independent random variables. Finally, the following conjecture has been suggested: the energy spectra of chaotic quantum systems are characterized by pink noise $1 / f$, in contrast to the Brown noise $1 / f^{2}$ of regular systems [4].

The Madrid group has analytically proved its conjecture on the basis of the RMT [5]. Independently, Robnik has obtained the same result [6]: under the conditions $M \gg 1$ and $k / M \ll 1$, the averaged power spectrum of the energy-level statistic $\delta_{n}$ is given by

$$
\left\langle S_{M}(k)\right\rangle=\left\{\begin{array}{cc}
\frac{2}{\beta} \frac{M}{k} & \text { for chaotic systems } \\
\frac{M^{2}}{k^{2}} & \text { for integrable systems }
\end{array},\right.
$$

where $\beta$ depends on the symmetry of the Gaussian ensemble: $\beta=1$ for the Gaussian Ortogonal Ensemble (GOE), $\beta=2$ for the Gaussian Unitary Ensemble (GUE), and $\beta=4$ for the Gaussian Symplectic Ensemble (GSE). Thus, although the origin of $1 / f$ noise in many complex systems is still an unsolved problem [7], the origin of $1 / f$ noise in the spectral fluctuations of chaotic quantum systems has been easier to understand because of the mathematical tractability of RMT. It is important to stress that Eq. (5) is valid if the condition $k / M \ll 1$ is fullfilled. In fact, as shown in [5] the Eq. (5) is the powerlaw approximation, obtained via Taylor expansion, of a more complex formula. In Fig. 1 we plot both the exact curve derived in [5] and the power-law curve of Eq. (5). Figure 1 shows that $\left\langle S_{M}(k)\right\rangle$ presents deviations from the power-law behavior for $k$ close to the Nyquist number $k_{c}=M / 2$. These deviations are due to the finite size $M$ of the series $\delta_{n}$ and the figure shows that they are negligible for $k / M \leq 10^{-2}$, where the relative error 
is below $1 \%$. In practice, because of the log-log scale, the differences are clearly visible only for $k / M>10^{-1}$.
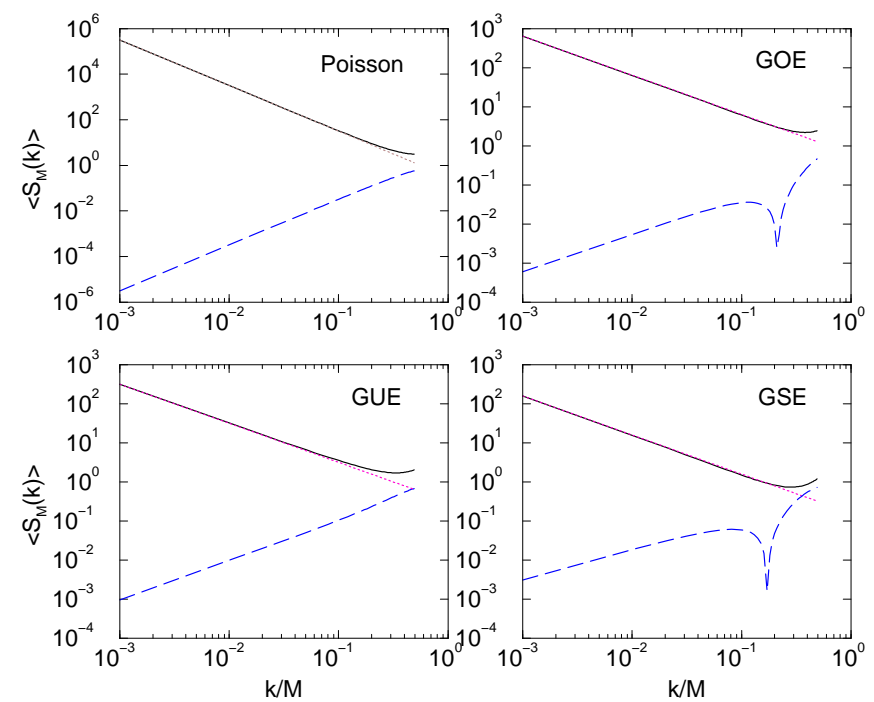

FIG. 1 (color online). Average power spectrum $\left\langle S_{M}(k)\right\rangle$ of the spectral statistic $\delta_{n}$. Comparison between the exact formula of Ref. [5] (solid line) and its power-law approximation (dotted line), given by Eq. (5). The dashed line is the relative error of the two curves. Note the different scale of the Poisson ensemble (integrable systems) with respect to the Gaussian ensembles (chaotic systems).

The proof of the nice result of Eq. (5) is based on the relationship between $S_{M}(k)$ and the widely studied spectral form factor of the density of levels $[5,6]$. Here, we apply the same methodology of Ref. $[5,6]$ to introduce and analyze a new set of spectral statistics, which contains $\delta_{n}$ as a particular case. First we define the following set of functions

$$
N_{o s c}^{(\alpha)}(E)=\frac{d^{\alpha} N_{o s c}(E)}{d E^{\alpha}},
$$

where the parameter $\alpha$ is a positive integer. This family of functions can be extended also to negative integer values of $\alpha$ by setting

$N_{o s c}^{(-|\alpha|)}(E)=\int_{-\infty}^{E} d x_{1} \int_{-\infty}^{x_{1}} d x_{2} \ldots \int_{-\infty}^{x_{|\alpha|-1}} d x_{|\alpha|} N_{o s c}\left(x_{|\alpha|}\right)$.

For $\alpha=0$ one recovers the oscillating part $N_{\text {osc }}(E)$ of the staircase function, while for $\alpha=1$ one has the oscillating part $\rho_{\text {osc }}(E)$ of the density of levels. In analogy with Eq. (2) we now introduce the following set of spectral statistics:

$$
\delta_{n}^{(\alpha)}=N_{o s c}^{(\alpha)}\left(E_{n}\right)
$$

spanned by the integer number $\alpha$. Obviously, for $\alpha=0$ one has the spectral statistic $\delta_{n}$ of Eq. (2).
By applying the formula of integration by parts, it is straightforward to prove that the Fourier transform of $N_{o s c}(E)$, given by

$$
\hat{N}_{o s c}(t)=\int_{-\infty}^{+\infty} N_{o s c}(E) \exp (-i E t) d E,
$$

is related to the Fourier transform of $N_{o s c}^{(\alpha)}(E)$ by the simple formula

$$
\hat{N}_{\text {osc }}(t)=\hat{N}_{\text {osc }}^{(\alpha)}(t) \frac{1}{(i t)^{\alpha}} .
$$

Under the conditions $M \gg 1$ and $k / M \ll 1$ the discrete Fourier transform of $\delta_{n}$ can be obtained from the Fourier transform of $N_{o s c}(E)$. It is given by

$$
\hat{\delta}_{k}=\hat{N}_{o s c}\left(\frac{k}{M}\right) .
$$

Taking into account Eqs. (3), (8), (10) and (11), the relationship between the power spectrum of $\delta_{n}$ and the power spectrum of $\delta_{n}^{(\alpha)}$ reads

$$
S_{M}(k)=\frac{S_{M}^{(\alpha)}(k) M^{2 \alpha}}{k^{2 \alpha}} .
$$

Finally, by using the Eq. (5) one finds that the average power spectrum of the spectral statistic $\delta_{n}^{(\alpha)}$ satisfies the following formula

$$
\left\langle S_{M}^{(\alpha)}(k)\right\rangle=\left\{\begin{array}{cc}
\frac{2}{\beta} \frac{M^{1-2 \alpha}}{k^{1-2 \alpha}} & \text { for chaotic systems } \\
\frac{M^{2-2 \alpha}}{k^{2-2 \alpha}} & \text { for integrable systems }
\end{array}\right.
$$

This formula is the main result of the paper. For $\alpha=0$ it reduces to Eq. (5). In general, for $\alpha>0$ the average power spectrum of $\delta_{n}^{(\alpha)}$ is not divergent, it is instead divergent for $\alpha \leq 0$.

Among the new spectral statistics, for numerical purposes the most simple ones are $\delta_{n}^{(-1)}$ and $\delta_{n}^{(1)}$. The statistic $\delta_{n}^{(-1)}$ is given by

$$
\delta_{n}^{(-1)}=\int_{-\infty}^{E_{n}} N_{o s c}(E) d E,
$$

and its average power spectrum is

$$
\left\langle S_{M}^{(-1)}(k)\right\rangle=\left\{\begin{array}{cc}
\frac{2}{\beta} \frac{M^{3}}{k^{3}} & \text { for chaotic systems } \\
\frac{M^{4}}{k^{4}} & \text { for integrable systems }
\end{array}\right.
$$

Thus, $\delta_{n}^{(-1)}$ shows black noise $1 / f^{3}$ for chaotic systems and black noise $1 / f^{4}$ for regular systems. The statistic $\delta_{n}^{(1)}$ is instead given by

$$
\delta_{n}^{(1)}=\frac{d N_{o s c}\left(E_{n}\right)}{d E}=\rho_{o s c}\left(E_{n}\right) .
$$


Its average power spectrum is nothing else than the discrete spectral form factor of the density of levels, which shows blue noise $f$ for chaotic systems and white noise $f^{0}=1$ for regular systems.
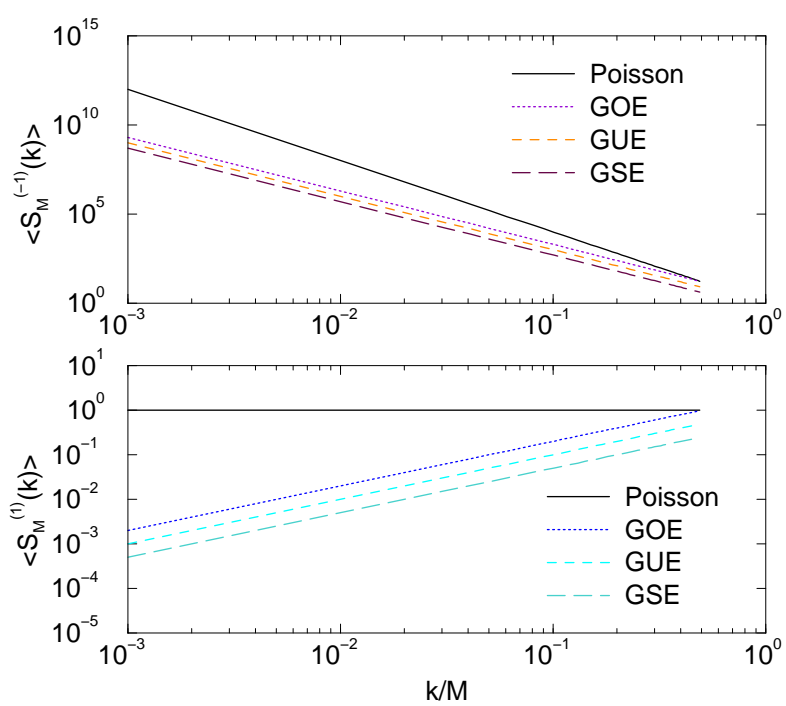

FIG. 2 (color online). Average power spectrum $\left\langle S_{M}^{(\alpha)}(k)\right\rangle$ of the spectral statistics $\delta_{n}^{(-1)}$ and $\delta_{n}^{(1)}$. Power laws for integrable systems (Poisson) and for chaotic systems with different classes of symmetry: GOE, GUE, and GSE.

For the sake of completeness, in Fig. 2 we plot the power spectrum of spectral statistics $\delta_{n}^{(-1)}$ (upper panel) and $\delta_{n}^{(1)}$ (lower panel). Figure 2 shows that different power laws can be easily distinguished by choosing the appropriate scale interval in the log-log plot. The new spectral statistics we have introduced are a simple and useful tool to analyze the energy levels of quantum systems and determine their integrability or chaoticity.

An open problem is the behavior of $\left\langle S_{M}^{(\alpha)}(k)\right\rangle$ for systems in the mixed regime between order and chaos. Recently we have analyzed the order to chaos transition in terms of the power spectrum $\left\langle S_{M}(k)\right\rangle$ by using the Pascal's snail (Robnik billiard) [8]. We have numerically found a net power law $1 / f^{\gamma}$, with $1 \leq \gamma \leq 2$, at all the transition stages. We have suggested that the exponent $\gamma$ is related to the chaotic component of the classical phase space of the quantum billiard, but a theoretical explanation of these numerical results is still lacking [9].

In conclusion, we have shown that colored noise $1 / f^{\gamma}$ characterizes the spectral fluctuations of quantum systems. The presence of colored noise in fluctuating physical variables is ubiquitous. This kind of noise has been detected in DNA sequences, quasar emission, river discharge, heartbeat, among many others. Despite this ubiquity, there is no universal explanation about this phenomenon, which is a generic manifestation of complex systems. Nevertheless, here we have shown that for integrable and chaotic quantum systems the colored noise of energy-level fluctuations can be explained in a satisfactory way on the basis of random matrix theory.

The author thanks J.M.G. Gomez, A. Relano, E. Faleiro and M. Robnik for many useful discussions.

[1] H.-J. Stöckmann, Quantum Chaos (Cambridge University Press, Cambridge, 1999).

[2] M.V. Berry and M. Tabor, Proc. R. Soc. London A 356, 375 (1977).

[3] O. Bohigas, M.J. Giannoni, and C. Schmit, Phys. Rev. Lett. 52, 1 (1984)

[4] A. Relano, J.M.G. Gomez, R.A. Molina, J. Retamosa, E. Faleiro, Phys. Rev. Lett. 89, 244102 (2002).

[5] E. Faleiro, J.M.G. Gomez, R.A. Molina, L. Munoz, A. Relano, and J. Retamosa, Phys. Rev. Lett. 93, 244101 (2004).

[6] M. Robnik, preprint CAMTP/2 December 2003; M. Robnik, preprint CAMTP/20 May 2004, submitted to International Journal of Bifurcation and Chaos.

[7] B.B. Mandelbrot, Multifractals and $1 / f$ noise (Springer, New York, 1999).

[8] M. Robnik, J. Phys. A: Math. Gen. 31, 3971 (1983); T. Prosen and M. Robnik, J. Phys. A: Math. Gen. 26, 2371 (1993).

[9] J.M.G. Gomez, A. Relano, J. Retamosa, E. Faleiro, L. Salasnich, M. Vranicar, and M. Robnik, condmat/0502130, accepted for publication in Phys. Rev. Lett. 